\title{
Robust optimization and uncertainty quantification in the nonlinear mechanics of an elevator brake system
}

\author{
Piotr Wolszczak (D) P Pawel Lonkwic • Americo Cunha Jr. • Grzegorz Litak • \\ Szymon Molski
}

Received: 17 November 2018/Accepted: 4 May 2019/Published online: 14 May 2019

(C) The Author(s) 2019

\begin{abstract}
This paper deals with nonlinear mechanics of an elevator brake system subjected to uncertainties. A deterministic model that relates the braking force with uncertain parameters is deduced from mechanical equilibrium conditions. In order to take into account parameters variabilities, a parametric probabilistic approach is employed. In this stochastic formalism, the uncertain parameters are modeled as random variables, with distributions specified by the maximum entropy principle. The uncertainties are
\end{abstract}

P. Wolszczak $(\bowtie) \cdot$ G. Litak

Faculty of Mechanical Engineering, Lublin University of Technology, Nadbystrzycka 36, 20-618 Lublin, Poland e-mail: p.wolszczak@pollub.pl

G. Litak

e-mail: g.litak@pollub.pl

P. Lonkwic

Mechanical Engineering Faculty, State School of Higher Education, Pocztowa 54, 22-100 Chelm, Poland e-mail: plonkwic@gmail.com

A. Cunha Jr.

Nucleus of Modeling and Experimentation with Computers - NUMERICO, Universidade do Estado do Rio de Janeiro, Rua São Francisco Xavier, 524,

Rio de Janeiro, RJ 20550-900, Brazil

e-mail: americo@ime.uerj.br

S. Molski

Department of Rope Transport, AGH University of

Science and Technology, Mickiewicza 30,

30-059 Kraków, Poland

e-mail: molski@agh.edu.pl propagated by the Monte Carlo method, which provides a detailed statistical characterization of the response. This work still considers the optimum design of the brake system, formulating and solving nonlinear optimization problems, with and without the uncertainties effects.

Keywords Elevator brake system · Nonlinear mechanics · Nonlinear optimization · Uncertainty quantification $\cdot$ Parametric probabilistic approach

\section{Introduction}

Considerations regarding the construction of lifting devices (design of cranes), and in particular brake systems, are not often discussed in the scientific literature, as the corresponding dynamical conditions are difficult to determine. The first person who addressed the issue of the impact of safety gears construction on the braking distance was Elisha Graves Otis, who in 1853 built the first safety gears and subjected them to experimental studies [1, 2]. Subsequent works on this subject, studying several aspects of cranes mechanics, appeared in the twentieth century and were published in journals and conference proceedings [3-9].

For instance, Yost and Rothenfluth [3] describe how to configure a lifting device and how to select the correct components. These issues constituted a 
significant contribution to the development of the configuration of lifts, ensuring a trouble-free operation.

Lonkwic [4] presents a comparative analysis of the operation of slip safety gear of his own design study with the models by leading European manufacturers. Deceleration (braking time) values obtained in the physical experiment are analyzed. In [5], the same author and collaborators address, by means of wavelet analysis, how certain variables influence on the operating conditions of deceleration. A similar analysis is presented in [6], which concerns the selected braking parameters of CHP2000 and PP16 type chaters using the analysis of recursive patterns.

Regarding the study of elevator systems with uncertain operating conditions, the literature is not very comprehensive. The only works in this line known by the authors are [7, 8], developed by Kaczmarczyk et al., who attempt to analyze the behavior of balance ropes due to harmonic and stochastic excitations, and Colón et al. [9], who calculate the propagation of the rail profile uncertainties and study the effectiveness of a closed-loop control law.

Even with the scientific literature being rich in studies regarding the behavior of vehicles brake systems under changing operating conditions [10-12], it is surprising that, to the best of authors' knowledge, no similar research description on lift brakes has been reported up to the present date. Only general provisions contained in the British Standard Document BS EN $81[13,14]$ are to be found.

Thus, seeking to fill this gap, the present work aims to study the influence of some operating conditions on the efficiency of an elevator brake device, by analyzing how the operating parameters underlying uncertainties propagate through the mechanical system. In particular, the cam brake angle and the spring reaction force are of interest. In addition to quantifying the effects of uncertainties in operating conditions, this study also aims to achieve a robust design of a brake system by solving a nonlinear optimization problem, considering (or not) the uncertainty effects.

The remaining part of this paper is organized as follows: the deterministic modeling of the lift brake systems under study is presented in Sect. 2. In Sect. 3, the construction of a consistent stochastic model of uncertainties to deal with variabilities in the uncertain parameters, is presented. Two optimization problems, one classical and one robust, which seek to find an optimal design for the brake system are formulated in Sect. 4. In Sect. 5, numerical experiments are reported and discussed. Finally, in Sect. 6, concluding remarks are presented.

\section{Deterministic modeling}

\subsection{Elevator brake system}

A schematic representation of the CHP 2000 safety gear, used by a typical friction crane brake system, is presented in Fig. 1. It consists of a monolithic steel body (1), in which a braking cam (5) is mounted on a bolt. The braking cam moves the brake roller (2), which has a knurled surface. This irregular surface is responsible for the braking process and for the cooperation with the guide roller surface. The brake roller moves over the braking cam surface until it contacts the lift guide (6). It is a free movement that does not cause any braking effect. The second part of

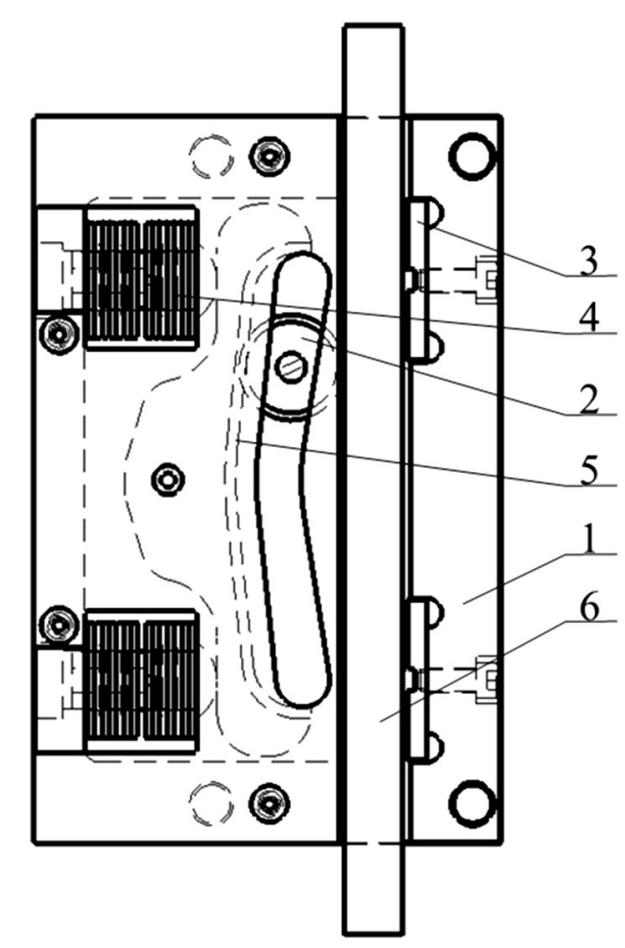

Fig. 1 Schematic representation of the CHP 2000 safety gear used by a friction crane brake system, which consists of the following parts: 1 -steel body; 2-brake roller; 3 -thrust plate; 4-spring package; 5-braking cam; 6-lift guide 
the braking process is in constant contact with the lift guide surface.

An illustration of a typical friction crane brake system used by lifting devices is shown in Fig. 2, which indicates the different components of the mechanism (see the caption). It consists of two safety gears, moving on the lift guides, connected to each other to ensure simultaneous operation when the brakes are activated by means of a trigger lever. A lift safety gear is placed in the frame, under a safety gear cabin. Its trigger is attached to the trigger lever, in which the end is connected to the rope speed limiter. In the upper part of the elevator shaft there is a speed limiter supervising the work of the safety gear, and in its lower part load responsible for causing the proper tension of the speed limiter rope is located. The speed limiter triggers the braking process when the nominal speed of the elevator car is increased by $0.3 \mathrm{~m} / \mathrm{s}$. After exceeding the nominal speed, the speed limiter is blocked, and the rope is also immobilized.

During the movement of an elevator car with locked components, the lever is moved in the opposite direction to the cabin, triggering the brake safety gear roll. In its turn, the roll is pressed against the guide causing elastic deformation towards the thrust plate located on the other side of the disk spring package, which induces the loss of energy in the accelerating mass. Therefore, the disc spring package is responsible

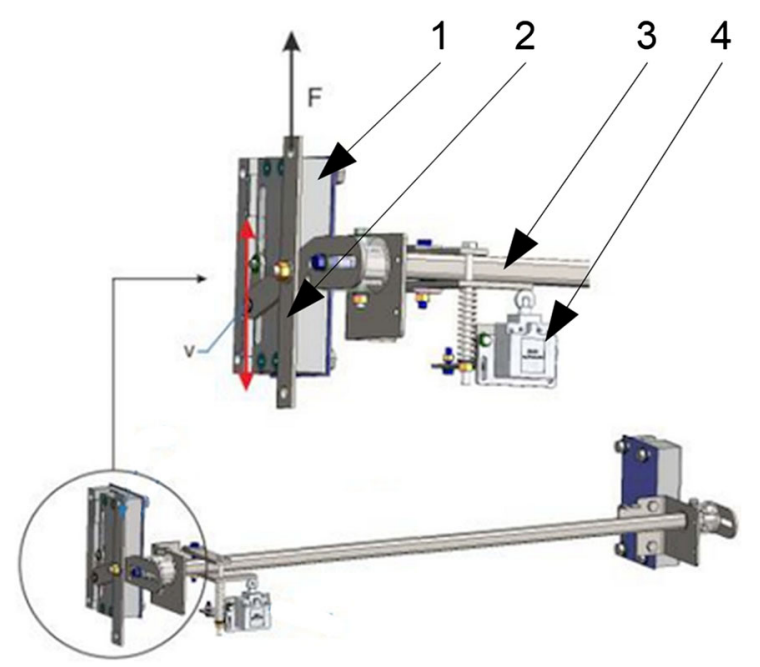

Fig. 2 Illustration of a typical friction crane brake system used by lifting devices, which consists of the following parts: $1-$ safety gear cabin; 2-trigger lever; 3-safety gears connector; 4-rope speed limiter for a variable force that presses the roller to the guide during the braking process.

\subsection{Mathematical model}

The design assumptions and safety gear structure shown in Fig. 1 are taken into account to construct a mathematical model that relates the braking force with geometric parameters and other characteristics of the mechanical system. In this sense, equilibrium conditions for the system are deduced below.

A free-body diagram can be seen in Fig. 3, which shows a schematic representation of the forces (in red) acting on the safety gear steel body, and the underlying geometric dimensions (in blue).

A balance of forces and the moments acting on the steel body gives rise to the equations

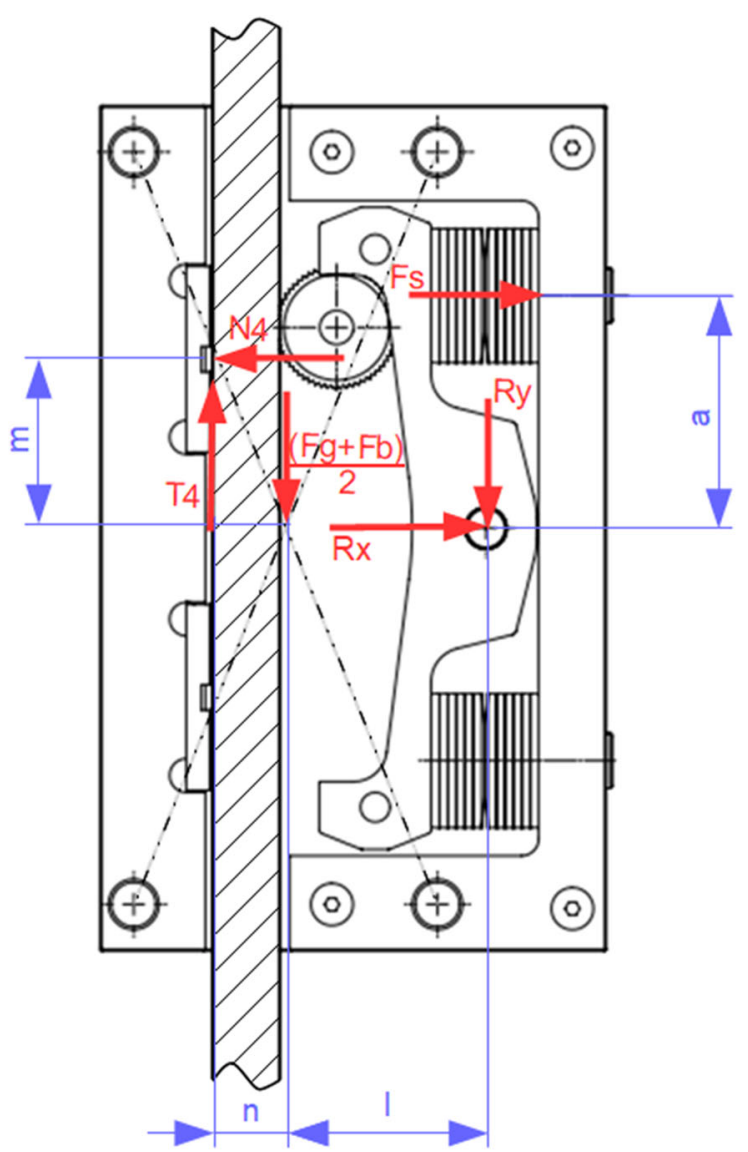

Fig. 3 Illustration of the forces (in red) acting on the safety gear steel body and the underlying geometric dimensions (in blue). (Color figure online) 


$$
-N_{4}+F_{s}+R_{x}=0
$$

$T_{4}-R_{y}-\left(F_{g}+F_{b}\right) / 2=0$,

$$
-F_{s} a-R_{y} l+N_{4} m-T_{4} n=0,
$$

where $F_{s}$ is the spring reaction force; $F_{b}$ is the inertial force from the cabin and lifting capacity; $F_{g}$ is the cabin and lifting capacity weight; $T_{4}$ is the friction force between the guide and brake retaining block, and $N_{4}$ is the corresponding normal force; $R_{x}$ and $R_{y}$ are the reaction forces in the braking cam rotation point; while $a, l, m$ and $n$ are geometric dimensions depicted in Fig. 3.

The forces (in red) acting on the wedge during braking and immediately after stopping the cabin, until the safety gears are unlocked by technical maintenance of the lift, are shown in Fig. 4, along with the relevant geometric dimensions (in blue).

A new balance of forces and moments provides

$$
\begin{aligned}
& T_{2}+N_{1} \cos \alpha+T_{1} \sin \alpha-F_{s}-R_{x}=0, \\
& F_{s} a+T_{1} b-N_{1} c-N_{2} d-T_{2} e=0,
\end{aligned}
$$

where $T_{1}$ and $T_{2}$ are friction forces between brake elements (roller and cam), $N_{1}$ and $N_{2}$ are the

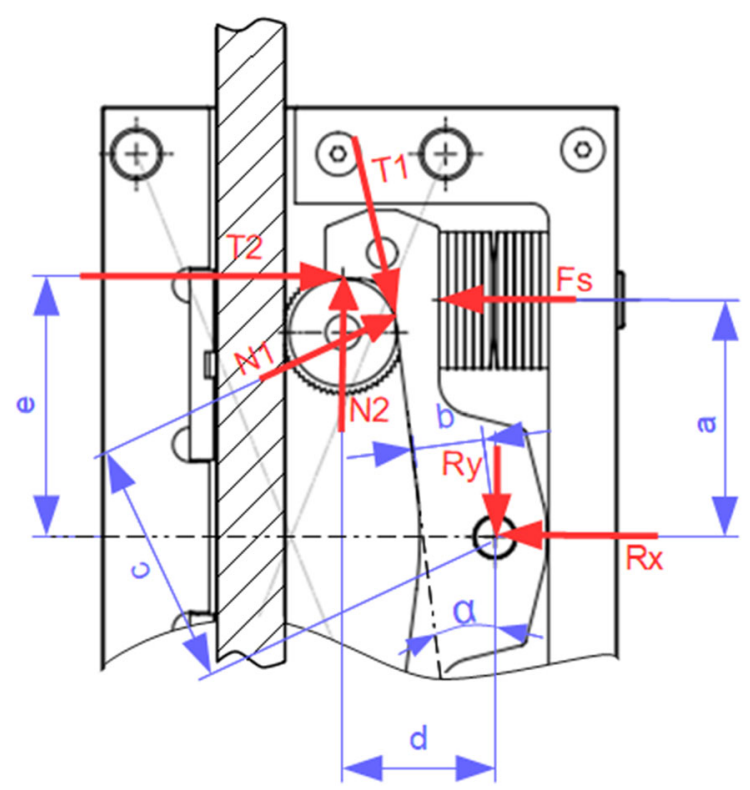

Fig. 4 Illustration of the forces (in red) acting on the wedge and the underlying geometric dimensions (in blue). (Color figure online) corresponding normal forces; $\alpha$ is the braking cam angle; $b, c, d$ and $e$ are other geometric dimensions of the problem, shown in Fig. 4.

In Fig. 5 the reader can see characteristic dimensions (in blue) and forces (in red) acting on the brake roller inside the safety gear.

Now the balance of forces gives

$N_{3}-T_{2}-N_{1} \cos \alpha-T_{1} \sin \alpha=0$,

$T_{3}-N_{2}+T_{1} \cos \alpha-N_{1} \sin \alpha=0$,

where $T_{3}$ and $N_{3}$ respectively denotes the frictional and the normal forces between brake roller and the guide.

The frictional forces $T_{1}, T_{2}$ and $T_{4}$ are, respectively, related to the normals $N_{1}, N_{2}$ and $N_{4}$ through a Coulomb friction model, so that

$T_{1}=\mu_{1} N_{1}$,

$T_{2}=\mu_{2} N_{2}$

$T_{4}=\mu_{4} N_{4}$,

where $\mu_{1}, \mu_{2}$ and $\mu_{4}$ are friction coefficients.

On the other hand, the relationship between the frictional force $T_{3}$ and the normal $N_{3}$ takes into account the plastic deformation occurring in the contact between brake roller and the guide, so that

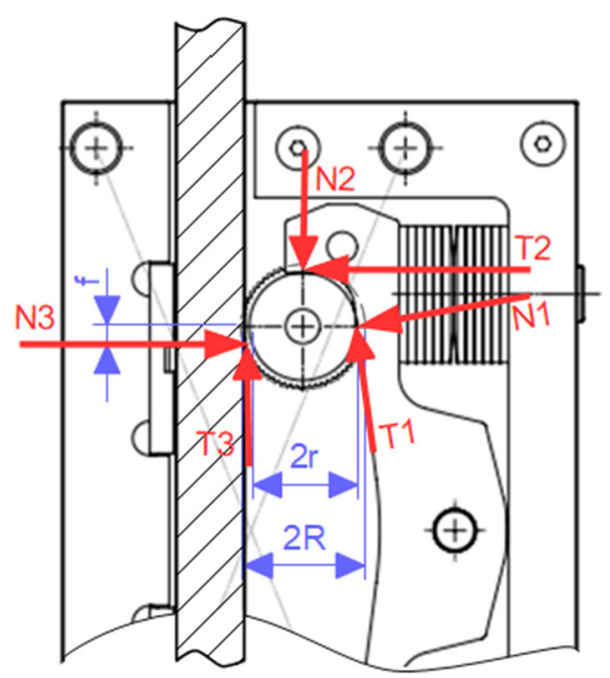

Fig. 5 Illustration of the forces (in red) acting on the brake roller inside the safety gear and and the underlying geometric dimensions (in blue). (Color figure online) 


$$
T_{3}=\frac{f}{R} N_{3},
$$

where $f$ and $R$ are geometric dimensions defined in Fig. 5.

The vertical reaction force $R_{y}$ can be obtained from Eqs. (2) and (3),

$$
\begin{aligned}
& R_{y}=-T_{4}+\frac{\left(F_{g}+F_{b}\right)}{2}, \\
& R_{y}=\frac{F_{s} a-N_{4} m+T_{4} n}{l},
\end{aligned}
$$

which, when combined together with Eq. (10), allows one to express $N_{4}$ as

$$
N_{4}=\frac{\left(F_{g}+F_{b}\right) l / 2-F_{s} a}{\mu_{4}(n+l)-m} .
$$

Similarly, from suitable manipulations of Eqs. (6), (8) and (9), it can be concluded that

$$
N_{3}=\mu_{2} N_{2}+\left(\mu_{1} \sin \alpha+\cos \alpha\right) N_{1},
$$

as well as, from Eqs. (5), (8) and (9), it is possible to obtain

$$
N_{2}=\frac{a F_{s}+\left(b \mu_{1}-c\right) N_{1}}{d+e \mu_{2}},
$$

which, in combination with Eqs. (1), (4), (8) and (9), gives rise to

$$
N_{1}=\frac{N_{4}-\frac{a \mu_{2} F_{s}}{d+e \mu_{2}}}{\mu_{1} \sin \alpha+\cos \alpha+\frac{\mu_{2}\left(b \mu_{1}-c\right)}{d+e \mu_{2}}} .
$$

The braking force, resulting from the joint superposition of all frictional forces, is given by

$$
F_{h}=T_{1}+T_{2}+T_{3}+T_{4},
$$

which, with aid of Eqs. (8)-(11), can be rewritten as

$$
F_{h}=\mu_{1} N_{1}+\mu_{2} N_{2}+\frac{f}{R} N_{3}+\mu_{4} N_{4} .
$$

Note that, once normal forces $N_{1}, N_{2}, N_{3}$ and $N_{4}$ present explicit dependence on geometric dimensions, frictional coefficients, and non-frictional forces, the braking force $F_{h}$ is also a function of these parameters, i.e.,

$$
\begin{gathered}
F_{h}=F_{h}\left(\alpha, F_{s}, F_{g}, F_{b}, \mu_{1}, \mu_{2}, \mu_{4}, \ldots\right. \\
\ldots a, b, c, d, e, f, l, m, n, R)
\end{gathered}
$$

\section{Stochastic modeling}

The angle $\alpha$ and the spring reaction force $F_{s}$ are subjected to variabilities during the operation conditions of the brake system, so that their actual values may be very different from the nominal project values. Since they are the critical parameters for the brake system efficiency, studying the effect of such variabilities on the braking force is essential for a good design. In this way, a parametric probabilistic approach $[15,16]$ is employed here to construct a consistent stochastic model for uncertain parameters $\alpha$ and $F_{s}$.

\subsection{Probabilistic framework}

Let $(\Theta, \Sigma, \mathcal{P})$ be the probability space used to describe the model parameters uncertainties [16, 17], where $\Theta$ is the sample space, $\Sigma$ a $\sigma$-field over $\Theta$, and $\mathcal{P}: \Sigma \rightarrow$ $[0,1]$ a probability measure.

In this probabilistic setting, the parameters $\alpha$ and $F_{s}$ are respectively described by the random variables $X_{1}: \Theta \rightarrow \mathbb{R}$ and $X_{2}: \Theta \rightarrow \mathbb{R}$, which are lumped into the random vector $X: \Theta \rightarrow \mathbb{R}^{2}$, which associates to each elementary event $\theta \in \Theta$ a vector $\boldsymbol{X}=\left(X_{1}, X_{2}\right)$. The probability distribution of $\boldsymbol{X}$ is characterized by the map $p_{X}: \mathbb{R}^{2} \rightarrow[0,+\infty)$, dubbed the joint probability density function (PDF).

The mean value of $\boldsymbol{X}$ is defined in terms of the expected value operator

$E\{\boldsymbol{X}\}=\iint_{\mathbb{R}^{2}} \boldsymbol{x} p_{\boldsymbol{X}}(\boldsymbol{x}) d \boldsymbol{x}$,

in which $\boldsymbol{x}=\left(x_{1}, x_{2}\right)$ and $d \boldsymbol{x}=d x_{1} d x_{2}$.

\subsection{Maximum entropy principle}

To perform a judicious process of uncertainty quantification, it is essential to construct a consistent stochastic model for the random vector $\boldsymbol{X}$, that represents the uncertainties in $\alpha$ and $F_{s}$ in a rational way, trying to be unbiased as possible. In this sense, in order to avoid possible physical inconsistencies in the 
probabilistic model, only available information must be used in its construction [16, 17]. When this information materializes in the form of a large set of experimental data, the standard procedure is to use a nonparametric statistical estimator to infer the joint distribution of $\boldsymbol{X}[16,17]$. However, if little (or even no) experimental data for $X_{1}$ and $X_{2}$ is available, as is the case of this paper, such construction can be done based only on known theoretical information, with the aid of the maximum entropy principle $[16,17]$.

The available theoretical information about the random parameters $X_{1}$ and $X_{2}$ encompasses a range of possible values for each of then, i.e.,

$$
\left(X_{1}, X_{2}\right) \in\left[\alpha_{1}, \alpha_{2}\right] \times\left[\gamma_{1}, \gamma_{2}\right] \subset[0,2 \pi] \times(0,+\infty),
$$

as well as their nominal values $\mu_{X_{1}}$ and $\mu_{X_{2}}$, that are assumed to be equal to their mean values, i.e.,

$$
\begin{aligned}
& E\left\{X_{1}\right\}=\mu_{X_{1}} \in\left[\alpha_{1}, \alpha_{2}\right], \\
& E\left\{X_{2}\right\}=\mu_{X_{2}} \in\left[\gamma_{1}, \gamma_{2}\right] .
\end{aligned}
$$

This information is translated into the statistical language through the normalization condition

$$
\int_{\gamma_{1}}^{\gamma_{2}} \int_{\alpha_{1}}^{\alpha_{2}} p_{X}(\boldsymbol{x}) d \boldsymbol{x}=1
$$

and the first order moment equation

$$
\int_{\gamma_{1}}^{\gamma_{2}} \int_{\alpha_{1}}^{\alpha_{2}} \boldsymbol{x} p_{\boldsymbol{X}}(\boldsymbol{x}) d \boldsymbol{x}=\left(\mu_{X_{1}}, \mu_{X_{2}}\right) .
$$

From the information theory point of view, the most rational approach to specify the distribution of $\boldsymbol{X}$ in this scenario of reduced information is through the maximum entropy principle (MaxEnt) [16-18], which seeks the PDF that maximizes the entropy functional

$$
S\left(p_{X}\right)=-\int_{\gamma_{1}}^{\gamma_{2}} \int_{\alpha_{1}}^{\alpha_{2}} p_{X}(\boldsymbol{x}) \ln p_{X}(\boldsymbol{x}) d \boldsymbol{x},
$$

respecting the restrictions (information) defined by (25) and (26).

Using the Lagrange multipliers method it is possible to show that such joint PDF is given by

$p_{\boldsymbol{X}}(\boldsymbol{x})=p_{X_{1}}\left(x_{1}\right) \times p_{X_{2}}\left(x_{2}\right)$,

with marginal densities
$p_{X_{1}}\left(x_{1}\right)=\exp \left(-\lambda_{10}-\lambda_{11} x_{1}\right) \mathbb{1}_{\left[\alpha_{1}, \alpha_{2}\right]}\left(x_{1}\right)$,

$p_{X_{2}}\left(x_{2}\right)=\exp \left(-\lambda_{20}-\lambda_{21} x_{2}\right) \mathbb{1}_{\left[\gamma_{1}, \gamma_{2}\right]}\left(x_{2}\right)$,

where $\lambda_{10}, \lambda_{11}, \lambda_{20}$ and $\lambda_{21}$ are parameters of the distribution of $\boldsymbol{X}$, and

$$
\mathbb{1}_{I}(x)= \begin{cases}1, & x \in I, \\ 0, & x \notin I,\end{cases}
$$

denotes the indicator function of the interval $I$. Note that, since no information relative to the cross statistical moments between $X_{1}$ and $X_{2}$ has been provided, MaxEnt provides independent distributions.

The parameters $\lambda_{10}, \lambda_{11}, \lambda_{20}$ and $\lambda_{21}$ depend on $\alpha_{1}$, $\alpha_{2}, \gamma_{1}, \gamma_{2}, \mu_{X_{1}}$ and $\mu_{X_{2}}$. They are computed through the nonlinear system of equations obtained by replacing (28) in (26) and in the normalization conditions of the marginal PDFs (29) and (30).

In a scenario with little information, it is practically impossible not to be biased in choosing a probability distribution. The MaxEnt formalism provides the least biased distribution that is consistent with the known information, therefore constituting the most rational approach [18].

\subsection{Uncertainty propagation}

The mathematical model relating the braking force $F_{h}$ with braking cam angle $\alpha$ and the spring reaction force $F_{s}$, Eq. (20), can be thought abstractly as a nonlinear deterministic functional $\mathcal{M}$ that maps a vector of input parameters $\boldsymbol{x}=\left(\alpha, F_{s}\right)$ into a scalar quantity of interest $y=F_{h}$, i.e.,

$\boldsymbol{x} \mapsto y=\mathcal{M}(\boldsymbol{x})$.

Thus, if the uncertain parameters $\alpha$ and $F_{s}$ are represented by the known random vector $\boldsymbol{X}$, the braking force becomes the random variable $Y=\mathcal{M}(\boldsymbol{X})$, for which the distribution must be estimated.

The process of determining the distribution of $Y$, once the probabilistic law of $\boldsymbol{X}$ is known, is dubbed uncertainty propagation problem $[15,16]$, being addressed in this paper via the Monte Carlo simulation [19, 20].

In this stochastic calculation technique, $v$ independent samples of $\boldsymbol{X}$ are drawn according to the density (28), giving rise to statistical realizations 


$$
\boldsymbol{X}^{(1)}, \boldsymbol{X}^{(2)}, \ldots, \boldsymbol{X}^{(v)}
$$

Each of these scenarios for $\boldsymbol{X}$ is given as input to the nonlinear deterministic map $\boldsymbol{x} \mapsto y=\mathcal{M}(\boldsymbol{x})$, resulting in a set of possible realizations for the quantity of interest

$$
Y^{(1)}, Y^{(2)}, \ldots, Y^{(v)},
$$

where $Y^{(j)}=\mathcal{M}\left(\boldsymbol{X}^{(j)}\right), j=1, \ldots, v$. These samples are used to estimate statistics of $Y$ non-parametrically, i.e., without assuming the PDF shape known [21].

\section{Optimization framework}

Regarding the improvement of brake system efficiency, an optimal design of its components is required. This work addresses this question by solving nonlinear optimization problems that seeks to maximize the braking force, using geometric dimensions of the system as design variables.

Two optimization approaches are employed. The first one, named classical, is based on deterministic formalism of nonlinear programming [22], while the latter, dubbed robust, takes into account the model parameters uncertainties, in order to reduce the optimum point sensitivity to small disturbances [23, 24].

In this framework, a set of two design variables (geometric dimensions) is denoted generically by the vector $s$. The other parameters of the model are denoted generically by $\boldsymbol{x}$, and the model response is given by the nonlinear map $(\boldsymbol{s}, \boldsymbol{x}) \mapsto y=\mathcal{M}(\boldsymbol{s}, \boldsymbol{x})$ The quantity of interest to be optimized (objective function) is denoted generically by $\mathcal{J}$.

\subsection{Classical optimization}

In this classical optimization approach the $s$ components are employed as design variables, while the braking force is adopted as objective function, i.e.,

$$
\mathcal{J}_{C}(\boldsymbol{s})=y \text {. }
$$

The admissible set for this optimization problem is defined by $\mathcal{A}_{C}=\left[s_{1}^{\min }, s_{1}^{\max }\right] \times\left[s_{2}^{\min }, s_{2}^{\max }\right]$, so that it can be formally stated as find an optimal design vector
$\boldsymbol{s}_{C}^{\text {opt }}=\underset{\boldsymbol{s} \in \mathcal{A}_{C}}{\arg \max } \mathcal{J}_{C}(\boldsymbol{s})$.

\subsection{Robust optimization}

In this robust optimization framework, which is based on those shown in [25, 26], the uncertainties are described according to the formalism of the Sect. 3, where $\boldsymbol{x}$ becomes the random vector $\boldsymbol{X}$, and, as a consequence, $y=\mathcal{M}(\boldsymbol{s}, \boldsymbol{x})$ becomes the random variable $Y=\mathcal{M}(\boldsymbol{s}, \boldsymbol{X})$.

Thus, the robust objective function is not constructed directly from the model response, but with the aid of statistical measures of $Y$, which aims to guarantee greater stability to small disturbances (robustness) to an optimum point.

Specifically, the robust objective function is given by a convex combination between minimum, maximum, mean and standard deviation inverse, so that

$$
\begin{aligned}
\mathcal{J}_{R}(s)= & \beta_{1} \min _{\theta \in \Theta}\{Y\}+\beta_{2} \max _{\theta \in \Theta}\{Y\}+\beta_{3} E\{Y\} \\
& +\beta_{4} \frac{1}{\sqrt{E\left\{Y^{2}\right\}-E\{Y\}^{2}}},
\end{aligned}
$$

where $\beta_{1}+\beta_{2}+\beta_{3}+\beta_{4}=1$.

Note that by maximizing this robust objective function, it is sought to raise both the lowest and the highest possible value, the mean, in addition to reducing the dispersion, by reducing the standard deviation.

Additionally, in order to avoid excessively small braking forces, the following probabilistic constraint is imposed

$$
\mathcal{P}\left\{|Y|>y^{*}\right\} \geq 1-P_{r},
$$

where $y^{*}$ is a lower bound for the magnitude of $Y$, and $P_{r}$ is reference probability.

Therefore, the admissible set for the robust optimization problem, denoted by $\mathcal{A}_{R}$, is defined as the subset of $\mathcal{A}_{C}$ for which the probabilistic constraint (38) is respected. In this way, the robust optimization problem is formally defined as find an optimal design vector

$$
\boldsymbol{s}_{R}^{\text {opt }}=\underset{\boldsymbol{s} \in \mathcal{A}_{R}}{\arg \max } \mathcal{J}_{R}(\boldsymbol{s}) .
$$




\section{Results and discussion}

The simulations reported below, conducted in Matlab, use the following numerical values for the deterministic parameters of the mechanical model: $F_{g}=50$ $\mathrm{kN} ; F_{b}=30 \mathrm{kN} ; \mu_{1}=0.10 ; \mu_{2}=0.10 ; \mu_{4}=0.15$; $a=55.0 \mathrm{~mm} ; b=16.6 \mathrm{~mm} ; c=52.7 \mathrm{~mm} ; d=34.5$ $\mathrm{mm} ; e=60.7 \mathrm{~mm} ; f=0.005 \mathrm{~mm} ; l=49.0 \mathrm{~mm} ; m=$ $40.0 \mathrm{~mm} ; n=17.5 \mathrm{~mm} ; R=29.0 \mathrm{~mm}$.

Regarding the two random parameters, the following information is assumed: $\left[\alpha_{1}, \alpha_{2}\right]=[0,18]^{\circ}$; $\left[\gamma_{1}, \gamma_{2}\right]=[0,56] \mathrm{kN} ; \mu_{X_{1}}=6^{\circ}$; and $\mu_{X_{2}}=42 \mathrm{kN}$.

\subsection{Uncertainty quatification}

The calculation of the propagation of uncertainties of $\boldsymbol{X}=\left(X_{1}, X_{2}\right)$ through the mechanical-mathematical model (32) initially involves the generation of random samples according to the probabilistic model defined by Eq. (28). A set of 4096 random samples for $X_{1}$ (top) and $X_{2}$ (bottom) can be seen in Fig. 6, which also shows some statistics (mean, standard deviation and $95 \%$ confidence interval) for this set of values.

In Fig. 7 the reader can see the statistics shown in Fig. 6 compared to the analytical curves for the PDFs of $X_{1}$ and $X_{2}$, and histograms constructed with the underlying random samples. It can be observed that the sampling process is well conducted, since the histograms and analytical curves present great similarity.

Note that the brake cam angle $X_{1}$ is modeled according to a probability density with a descending exponential behavior, which decays slowly between the ends of the support $\left[\alpha_{1}, \alpha_{2}\right]=[0,18]^{\circ}$, whereas the spring reaction force $X_{2}$ is described by probabilistic law with an increasing exponential density, which grows rapidly from the left to the right extreme of $\left[\gamma_{1}, \gamma_{2}\right]=[0,56] \mathrm{kN}$.

It is worth noting that, of course, the real system parameters do not follow these probability distributions. These are only approximations of the real distributions, constructed with the aid of the maximum entropy principle and the available information about these parameters. However, as in this paper the authors do not have experimental data to infer the real form of these distributions, in the light of information theory, the PDFs of Fig. 7 are the best that can be inferred.
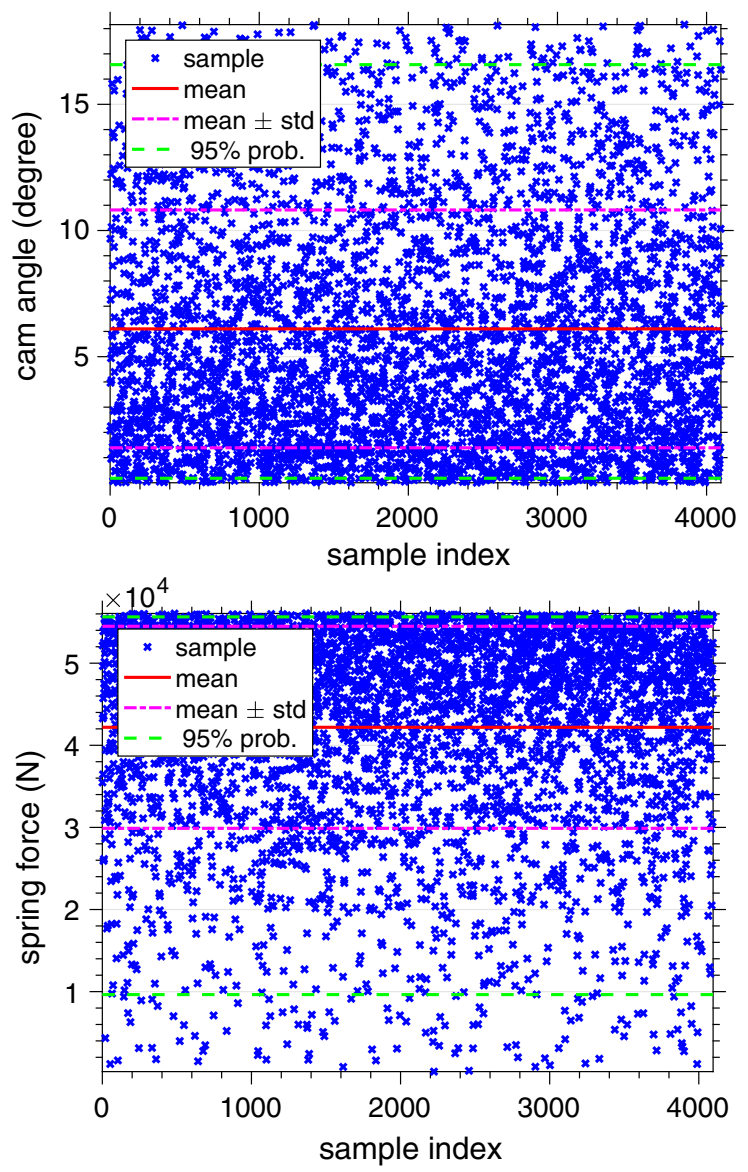

Fig. 6 Generated samples and statistical measures for the cam angle (top) and the spring force reaction (bottom)

The next step involves the model evaluation in each pair $\left(X_{1}, X_{2}\right)$ previously generated, which gives rise to the set of possible values for the braking force $F_{h}$, shown in the top part of Fig. 8. In the bottom part of the same figure the reader can observe a histogram that estimates the $F_{h}$ PDF form, as well as a nonparametric fitting obtained by a smooth curve. Mean, standard deviation, and a $95 \%$ confidence interval can be seen in both, top and bottom figures. To prove that these estimates are reliable, the authors also show the convergence of the mean and standard deviation estimators, as a function of the number of samples, in Fig. 9.

It may be noted that the PDFs of $F_{h}$ and $F_{s}$ have a very similar shape, suggesting that the mechanical model preserves the shape of the spring reaction force distribution. This result is at the least curious and unexpected, since the angular dependencies 

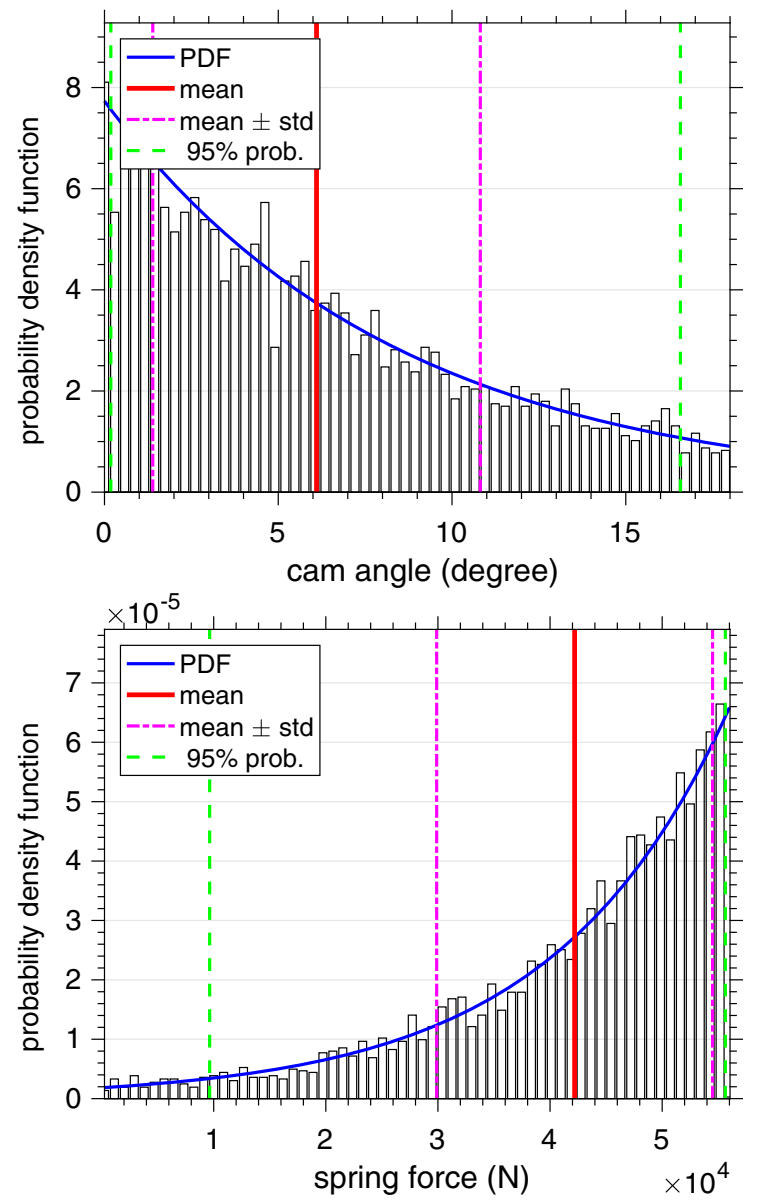

Fig. 7 Probability density functions and statistical measures for the cam angle (top) and the spring force reaction (bottom)

introduced in the mechanical model by Eqs. (15) and (17) define a structure of multiplicative uncertainty between $\alpha$ and $F_{s}$, what should make $F_{h}$ not invariant with respect to the input distribution.

This result suggests that the nonlinearity associated with the alpha parameter is very weak, which causes $F_{h}$ to behave as an affine map of $F_{s}$, and thus to preserve the form of its distribution. This hypothesis is reinforced by analyzing the system response by keeping $\alpha$ distribution and $F_{s}$ support fixed, while the mean value of the latter parameter is varied, assuming the values equal to for $14 \mathrm{kN}, 28 \mathrm{kN}^{1}$ and 42 $\mathrm{kN}$. The probability densities corresponding to these different inputs, and the corresponding outputs of the mechanical system can be seen in Figs. 10 and 11,

\footnotetext{
${ }^{1}$ For this value, which corresponds to the midpoint of the support, the distribution degenerates into an uniform.
}
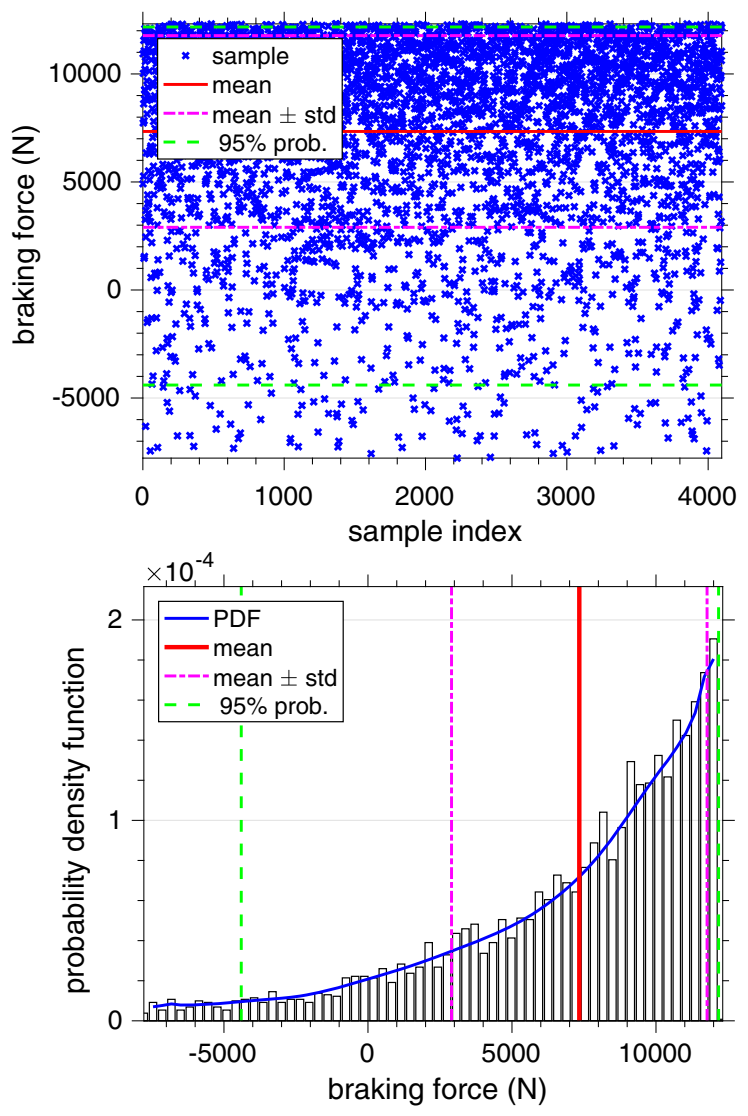

Fig. 8 Generated samples (top) and probability density function (bottom), with statistical measures, for the braking force

respectively. In all cases the input and output PDFs have the same shape.

The results of this study allow one to conclude that uncertainties in $\alpha$ parameter does not have significant influence on the braking force behavior. Simulations propagating only $\alpha$ uncertainties, not included here because of space limitation, demonstrate such an assertion. However, the uncertainty propagation study also shows that the variability of $F_{s}$ cannot be ignored, since it has great influence on the statistical behavior of $F_{h}$.

\subsection{Optimization}

In this section the problem of optimum design of the brake system is addressed. The geometric dimensions $\boldsymbol{s}=(a, c)$ are used as design variables, considering as admissible region $50 \leq a \leq 60 \mathrm{~mm}$ and $50 \leq c \leq 55$ $\mathrm{mm}$. 

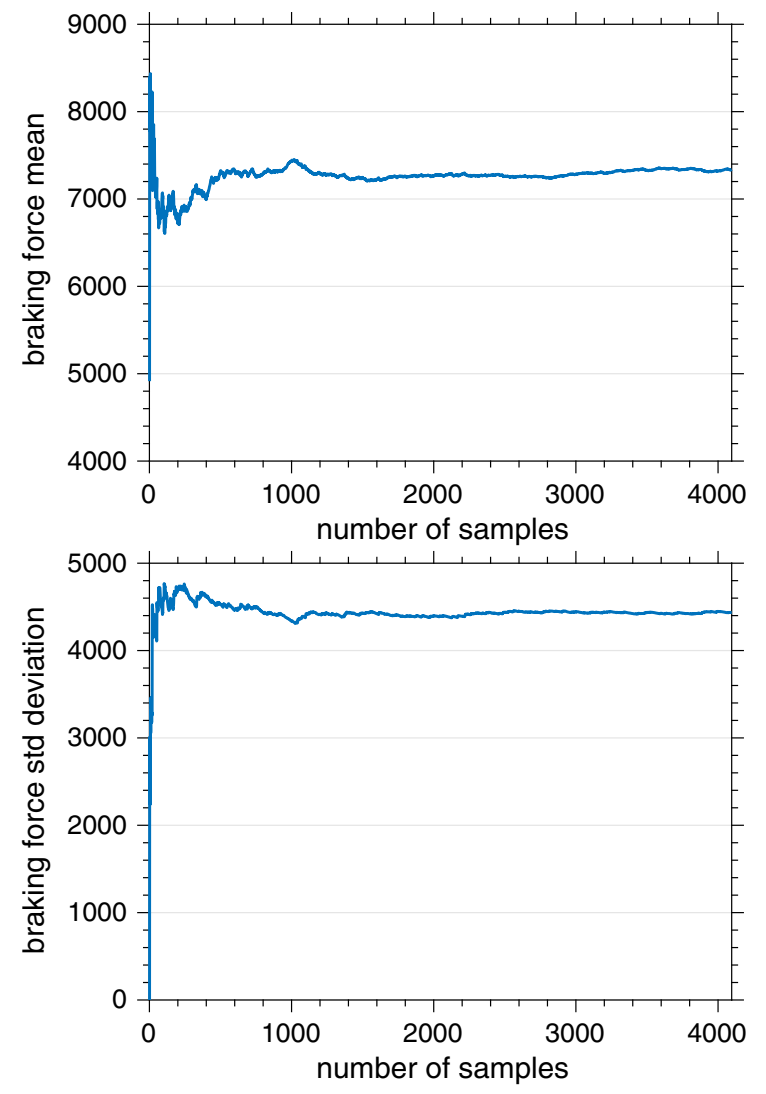

Fig. 9 Statistical estimators convergence for the braking force mean (top) and standard deviation (bottom)

The optimization problem is solved using the standard sequential programming quadratic (SQP) algorithm obtained from Matlab (see chap. 18 of [27]), being the contour map of the objective function (35) illustrated in Fig. 12, which also highlights the optimum point found.

Despite the fact that this result offers a starting point for an optimal project for the brake system, it does not take into account the effect of the uncertainties underlying the operating conditions, which can considerably affect the system response, as shown in the previous section. In this way, robust optimization presents itself as a natural alternative.

For the robust optimization problem the design variables $s=(a, c)$ are considered once more, with the same ranges of admissible values used above. The uncertainties in $\alpha$ and $F_{s}$ are modeled as in Sect. 3, and the probabilistic constraint is characterized by the parameters $y^{*}=0.5 \mathrm{kN}$ and $P_{r}=5 \%$. The convex weights $\beta_{1}=\beta_{2}=\beta_{3}=2 / 10$ and $\beta_{4}=4 / 10$ are adopted in the robust objective function.
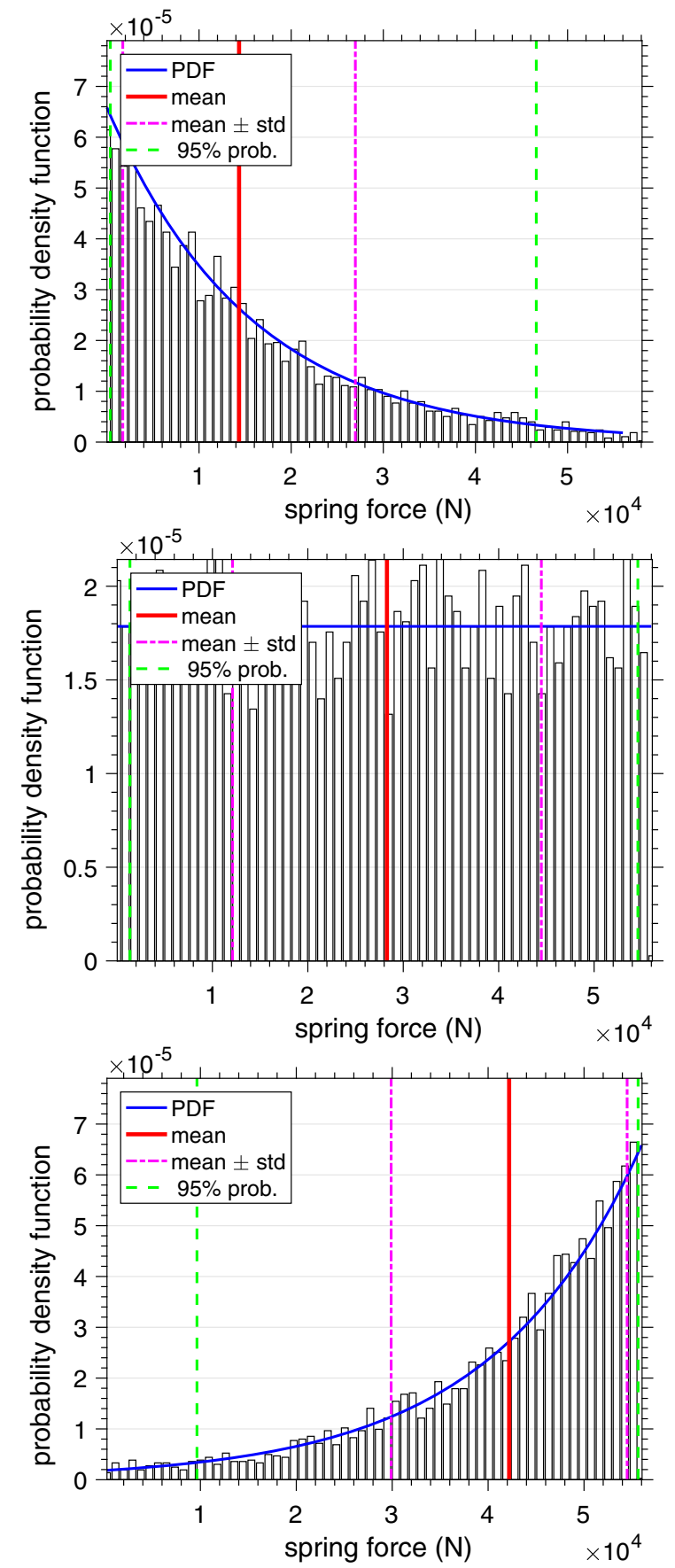

Fig. 10 Probability density functions and statistical measures for the spring force reaction with different mean values: $14 \mathrm{kN}$ (top); $28 \mathrm{kN}$ (middle); $42 \mathrm{kN}$ (bottom)

This second problem is much more complex because the constraint to be satisfied is nonconvex, offering additional challenges to the numerical 

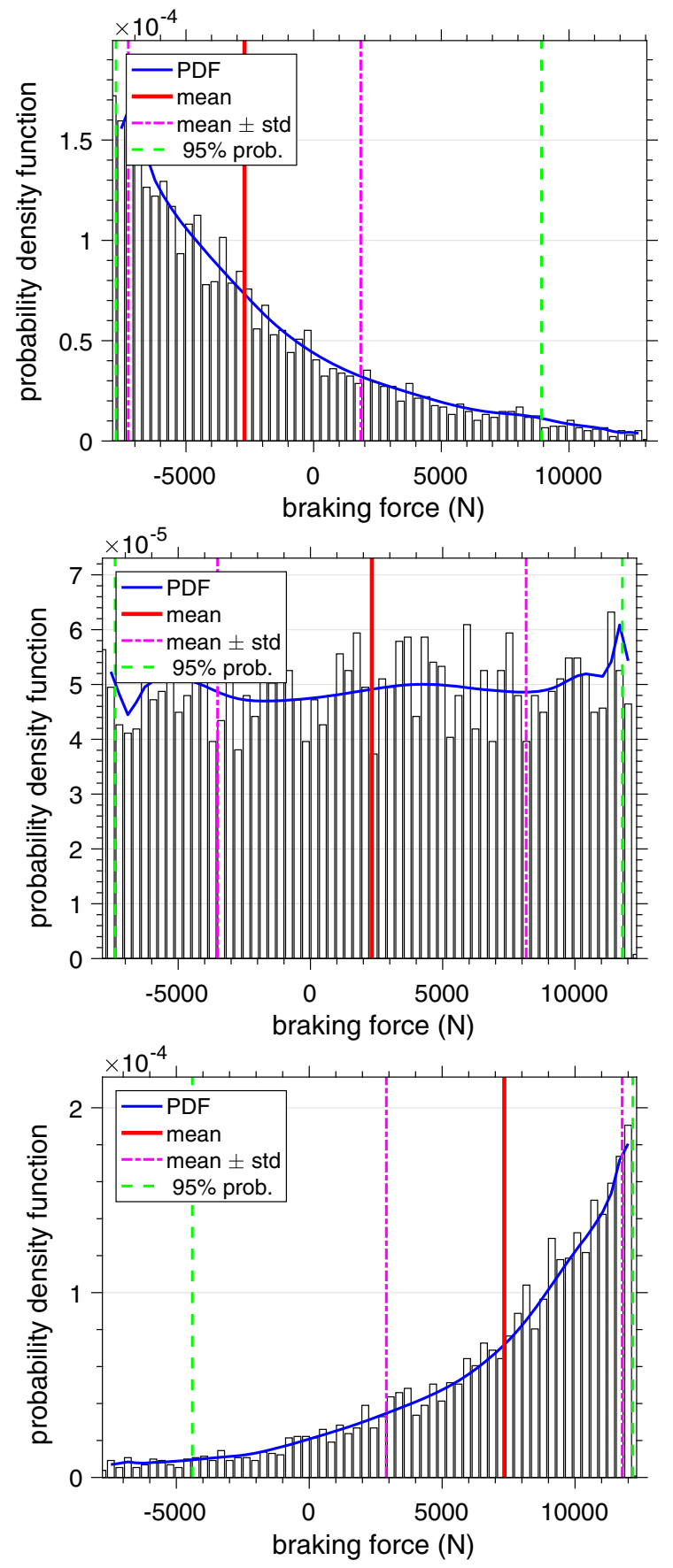

Fig. 11 Probability density functions and statistical measures for the braking force with different input mean values for $F_{s}: 14$ $\mathrm{kN}$ (top); $28 \mathrm{kN}$ (middle); $42 \mathrm{kN}$ (bottom)

solution procedure. But for the values described above the SPQ algorithm is able to find a solution.

The reader can see the contour map of the probabilistic constraint (38) in Fig. 13, while Fig. 14

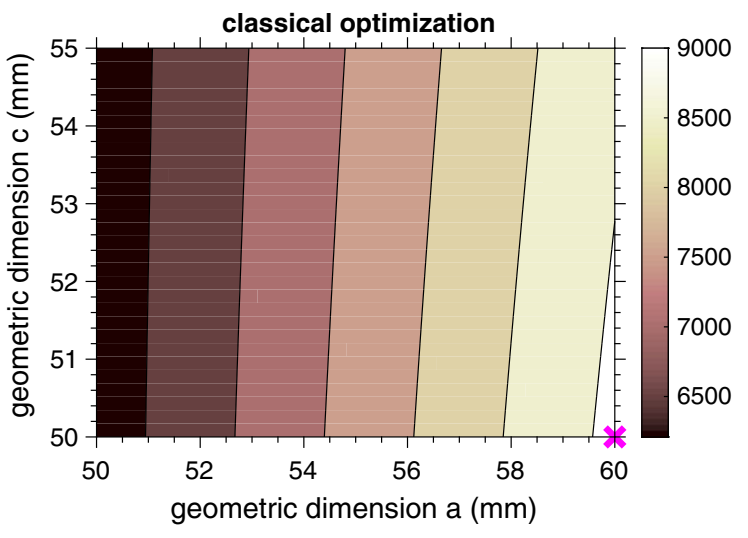

Fig. 12 Contour map for the classical objective function and with the optimum point is indicated by a cross

presents the contour map for the robust objective function (37). Although the objective function still maintains a smooth appearance, the problem gains a non-convex status by the irregular forms of constraint.

Once the robust objective function takes into account other design criteria than in (35), its behavior is different from the classical objective function shown in Fig. 12, thus having a different optimal point.

It is also worth noting that this second formulation of the optimization problem considers the effects of uncertainties, which in a realistic system are always present, thus offering a design option more suitable for projects that cannot ignore such variabilities.

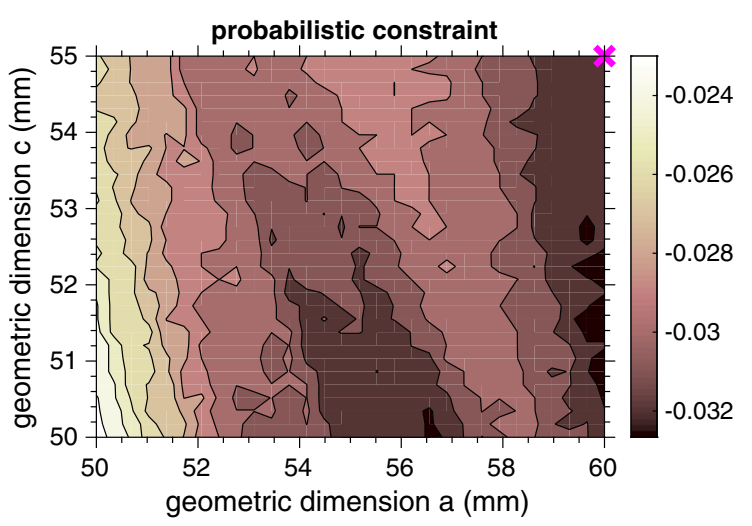

Fig. 13 Contour map for probabilistic constraint with the optimum point is indicated by a cross 


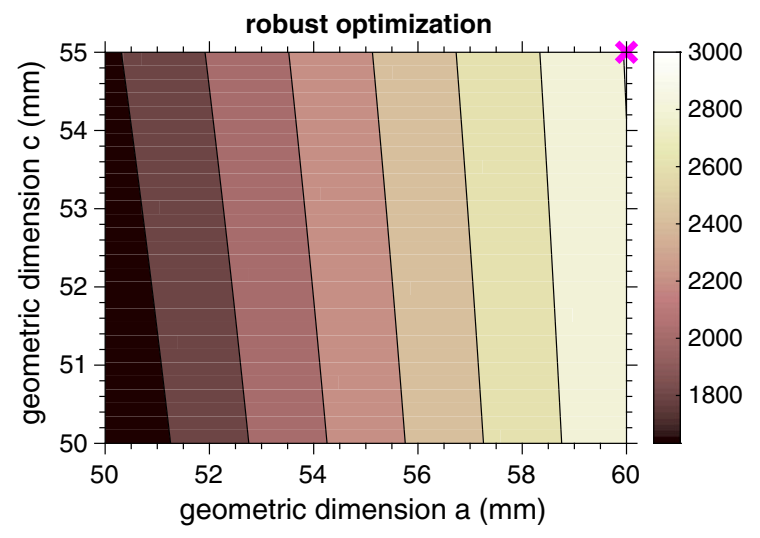

Fig. 14 Contour map for the robust objective function with the optimum point is indicated by a cross

\section{Summary and conclusions}

This work presents a study regarding the optimization and uncertainty quantification of an elevator brake system. The paper starts from an original construction of a safety gear for the brake, for which a mechanicalmathematical model is constructed. Studies involving the quantification of the braking force uncertainties due to the variability in the brake cam angle and the spring reaction force are presented, showing that spring force uncertainties are more significant. The paper also focuses on the optimal design of an elevator brake system, showing through the solution of a robust optimization problem that operating conditions uncertainties can significantly influence its efficiency.

Funding This research was financed in the framework of the project Lublin University of Technology-Regional Excellence Initiative, funded by the Polish Ministry of Science and Higher Education (Contract No. 030/RID/2018/19). The Grzegorz Litak acknowledge the financial support given by the Brazilian agencies Carlos Chagas Filho Research Foundation of Rio de Janeiro State (FAPERJ) under Grants E-26/ $010.002 .178 / 2015$ and E-26/010.000.805/2018 and Coordenação de Aperfeiçoamento de Pessoal de Nível Superior - Brasil (CAPES) - Finance Code 001.

\section{Compliance with ethical standards}

Conflict of interest The authors declare that they have no conflict of interest.

Open Access This article is distributed under the terms of the Creative Commons Attribution 4.0 International License (http:// creativecommons.org/licenses/by/4.0/), which permits unrestricted use, distribution, and reproduction in any medium, provided you give appropriate credit to the original author(s) and the source, provide a link to the Creative Commons license, and indicate if changes were made.

\section{References}

1. Lonkwic P (2017) Selected issues of the operating process of slide catches. Monography, Politechnika Lubelska, Lublin (in polish)

2. Pater Z (2011) Selected topics from the history of technology. Monography, Politechnika Lubelska, Lublin (in polish)

3. Yost GR, Rothenfluh TR (1996) Configuring elevator systems. Int J Hum Comput Stud 44:521-568. https://doi.org/ 10.1006/ijhc.1996.0023

4. Lonkwic P (2015) Influence of friction drive lift gears construction on the length of braking distance. Chin J Mech Eng 28:363-368. https://doi.org/10.3901/CJME.2015.0108. 009

5. Lonkwic P, Łygas K, Wolszczak P, Molski S, Litak G (2017) Braking deceleration variability of progressive safety gears using statistical and wavelet analyses. Measurement 110:90-97. https://doi.org/10.1016/j.measurement.2017.06. 005

6. Lonkwic P, Syta A (2016) Nonlinear analysis of braking delay dynamics for the progressive gears in variable operating conditions. J Vibroeng 18:4401-4408. https://doi.org/ $10.21595 /$ jve. 2016.17000

7. Kaczmarczyk S, Iwankiewicz R (2006) Dynamic response of an elevator car due to stochastic rail excitation. In: Proceedings of the Estonian Academy of Sciences, vol 55

8. Kaczmarczyk S, Iwankiewicz R, Terumichi Y (2009) The dynamic behaviour of a non-stationary elevator compensating rope system under harmonic and stochastic excitations. J Phys Conf Ser 181:012047. https://doi.org/10.1088/ 1742-6596/181/1/012047

9. Colón D, Cunha A Jr, Kaczmarczyk S, Balthazar JM (2017) On dynamic analysis and control of an elevator system using polynomial chaos and Karhunen-Loève approaches. Proc Eng 199:1629-1634. https://doi.org/10.1016/j.proeng.2017. 09.083

10. Renault A, Massa F, Lallemand B, Tison T (2016) Experimental investigations for uncertainty quantification in brake squeal analysis. J Sound Vibr 367:37-55. https://doi.org/10. 1016/j.jsv.2015.12.049

11. Dezi M, Forte P, Frendo F (2014) Motorcycle brake squeal: experimental and numerical investigation on a case study. Meccanica 49:1011-1021. https://doi.org/10.1007/s11012013-9848-y

12. Knops RJ, Villaggio P (2006) An optimum braking strategy. Meccanica 41:693-696. https://doi.org/10.1007/s11012006-9011-0

13. British Standards Institution (2018) Safety rules for the construction and installation of lifts: lifts for the transport of persons and goods-part 20: Passenger and goods passenger lifts. British Standards Institution, London

14. British Standards Institution (2018) Safety rules for the construction and installation of lifts: examinations and tests-part 50: Design rules, calculations, examinations and tests of lift components. British Standards Institution, London 
15. Cunha A Jr (2017) Modeling and quantification of physical systems uncertainties in a probabilistic framework. In: Ekwaro-Osire S, Goncalves AC, Alemayehu FM (eds) Probabilistic prognostics and health management of energy systems. Springer, New York, pp 127-156. https://doi.org/ 10.1007/978-3-319-55852-3_8

16. Soize C (2017) Uncertainty quantification: an accelerated course with advanced applications in computational engineering. Springer, New York. https://doi.org/10.1007/9783-319-54339-0

17. Soize C (2013) Stochastic modeling of uncertainties in computational structural dynamics: recent theoretical advances. J Sound Vibr 332:2379-2395. https://doi.org/10. 1016/j.jsv.2011.10.010

18. Kapur JN, Kesavan HK (1992) Entropy optimization principles with applications. Academic Press, Cambridge

19. Kroese DP, Taimre T, Botev ZI (2011) Handbook of Monte Carlo methods. Wiley, Hoboken

20. Cunha A Jr, Nasser R, Sampaio R, Lopes H, Breitman K (2014) Uncertainty quantification through Monte Carlo method in a cloud computing setting. Comput Phys Commun 185:1355-1363. https://doi.org/10.1016/j.cpc.2014. 01.006

21. Wasserman L (2007) All of nonparametric statistics. Springer, New York
22. Bonnans JF, Gilbert JC, Lemarechal C, SagastizÁbal CA (2009) Numerical optimization: theoretical and practical aspects, 2nd edn. Springer, New York

23. Beyer HG, Sendhoff B (2007) Robust optimization: a comprehensive survey. Comput Methods Appl Mech Eng 196:3190-3218. https://doi.org/10.1016/j.cma.2007.03.003

24. Gorissen BL, Yanikoglu I, den Hertog D (2015) A practical guide to robust optimization. Omega 53:124-137. https:// doi.org/10.1016/j.omega.2014.12.006

25. Cunha A Jr, Soize C, Sampaio R (2015) Computational modeling of the nonlinear stochastic dynamics of horizontal drillstrings. Comput Mech. https://doi.org/10.1007/s00466015-1206-6

26. Cuellar N, Pereira A, Menezes IFM, Cunha A Jr (2018) Non-intrusive polynomial chaos expansion for topology optimization using polygonal meshes. J Braz Soc Mech Sci Eng 40:561. https://doi.org/10.1007/s40430-018-1464-2

27. Nocedal J, Wright S (2006) Numerical optimization, 2nd edn. Springer, New York

Publisher's Note Springer Nature remains neutral with regard to jurisdictional claims in published maps and institutional affiliations. 